\title{
Modulation of an Afterhyperpolarization by the Substantia Nigra Induces Pauses in the Tonic Firing of Striatal Cholinergic Interneurons
}

\author{
John N. J. Reynolds, ${ }^{1,2}$ Brian I. Hyland, ${ }^{1,3}$ and Jeff R. Wickens ${ }^{1,2}$ \\ ${ }^{1}$ The Neuroscience Research Center and Departments of ${ }^{2}$ Anatomy and Structural Biology and ${ }^{3}$ Physiology, School of Medical Sciences, University of Otago, \\ Dunedin, New Zealand
}

\begin{abstract}
Striatal cholinergic interneurons, also known as tonically active neurons (TANs), acquire a pause in firing during learning of stimulusreward associations. This pause response to a sensory stimulus emerges after repeated pairing with a reward. The conditioned pause is dependent on dopamine from the substantia nigra, but its underlying cellular mechanism is unknown. Using in vivo intracellular recording, we found that both subthreshold and suprathreshold depolarizations in cholinergic interneurons induced a prolonged afterhyperpolarization (AHP) associated with a pause in their tonic firing. The AHP duration was dependent on the level of depolarization, whether elicited by intracellular current injection or by activation of excitatory inputs from the cortex. High-frequency stimulation of the substantia nigra induced potentiation of the cortically evoked excitation and increased the prolonged AHP after the stimulus. These findings from anesthetized animals suggest that a substantia nigra-induced AHP produces stimulus-associated firing pauses in cholinergic interneurons. This mechanism may underlie the acquisition of the pause response in TANs recorded from behaving animals during learning.
\end{abstract}

Key words: striatum; cholinergic; reward; pause; TAN; dopamine

\section{Introduction}

Recordings from striatal cells in behaving monkeys revealed a population of tonically active neurons (TANs) that acquire responsiveness to salient stimuli. The acquired response is a transient suppression of tonic firing or pause response, which is often preceded by an excitation (Kimura et al., 1984; Aosaki et al., 1994a). The maintenance of the pause response is dopaminedependent (Aosaki et al., 1994b; Watanabe and Kimura, 1998) and is thought to be important for learning because it appears and disappears in relationship to behavioral conditioning. However, little is known of the cellular mechanism of the pause response or of the processes underlying its development with conditioning.

Previous in vitro studies of striatal cholinergic interneurons (which correspond to the TANs) have led to two different hypotheses to explain the conditioned pause response. The first suggests that pauses are secondary to dopamine release. Bennett and Wilson (1998) showed that the stimulation of dopamine afferents prolongs the interval between successive spikes by enhancing the fast afterhyperpolarization (f-AHP) that follows individual action potentials. This requires activation of dopamine

Received Aug. 6, 2004; revised Sept. 2, 2004; accepted Sept. 4, 2004.

This work was supported by the Royal Society of New Zealand Marsden Fund, Lottery Health Research, and the Health Research Council of New Zealand. We thank Annabel Kean for technical assistance. This work is dedicated to our late colleague and friend Dr. Mark Tunstall.

Correspondence should be addressed to Prof. Jeff R. Wickens, Department of Anatomy and Structura Biology, University of Otago School of Medical Sciences, P.0. Box 913, Dunedin, New Zealand. E-mail: jeff.wickens@stonebow.otago.ac.nz.

D0I:10.1523/JNEUROSCI.3225-04.2004

Copyright $\odot 2004$ Society for Neuroscience $\quad 0270-6474 / 04 / 249870-08 \$ 15.00 / 0$
$\mathrm{D}_{1}$ receptors and is therefore consistent with the dopaminedependent maintenance of pause responses. However, against this hypothesis, spikes do not always precede the pauses in behaving animals (Apicella et al., 1991; Watanabe and Kimura, 1998), and pauses are maintained after fully predicted or aversive stimuli that minimally activate or even suppress dopamine neuron firing (Mirenowicz and Schultz, 1996; Ravel et al., 1999; Morris et al., 2004; Ungless et al., 2004).

The second hypothesis is that potentiation of inhibitory inputs may lead to an increased pause response. Suzuki et al. (2001) demonstrated, first, that high-frequency stimulation (HFS) of the corticostriatal or thalamostriatal pathway induced dopamine $\mathrm{D}_{1}$-like receptor-dependent potentiation of an EPSP in cholinergic interneurons. It has, however, been unclear how this would lead to firing pauses. Second, they demonstrated that a disynaptic IPSP appears more frequently after HFS, suggesting a possible basis for an increased pause after conditioning. However, the latency of inhibitory inputs is much shorter than that reported for the onset of a pause after a conditioned stimulus (Suzuki et al., 2001; Reynolds and Wickens, 2004). Thus, although likely to be involved in its acquisition, potentiation of IPSPs cannot account for all aspects of the pause response.

Here, we report a novel mechanism for the pause response based on potentiation of excitatory inputs to cholinergic interneurons. Our experiments involved in vivo intracellular recordings from intact anesthetized rats. Because of their rarity, very few cholinergic interneurons have ever been recorded using this technique (Bishop et al., 1982; Wilson et al., 1990). We obtained 
recordings from 14 cholinergic interneurons over a 6 year period while investigating synaptic responses in striatal neurons using standardized experimental procedures (Reynolds and Wickens, 2000, 2004). We report a novel prolonged AHP that can be evoked by membrane depolarization even in the absence of action potential firing. We further show that rewarding substantia nigra stimulation produces lasting changes in the prolonged AHP, which could account for the pause response seen in behavioral experiments.

\section{Materials and Methods}

Surgery. Male Wistar rats (280-400 gm) were anesthetized with urethane (1.4-1.9 gm/kg, i.p.; Biolab, Auckland, New Zealand) supplemented with ketamine $(10 \mathrm{mg} / \mathrm{kg}$, i.m.) and xylazine $(2 \mathrm{mg} / \mathrm{kg}$, i.m.; Southern Veterinary Supplies, Christchurch, New Zealand) or additional urethane $(0.2 \mathrm{gm} / \mathrm{kg})$ every $1-2 \mathrm{hr}$, as required. Stimulating electrodes were pairs of insect pins, insulated to within $0.5 \mathrm{~mm}$ of the tip and cemented to the skull overlying the medial agranular cortex contralateral to the recording site (anteroposterior, +10.6 to $+12.7 \mathrm{~mm}$; mediolateral, $-2.0 \mathrm{~mm}$ relative to interaural line; dorsoventral, $1.6-2.0 \mathrm{~mm}$ from the brain surface) and the ipsilateral substantia nigra pars compacta (anteroposterior, +3.5 to $+3.6 \mathrm{~mm}$; mediolateral, $+1.6 \mathrm{~mm}$ relative to interaural line; dorsoventral, 7.2-7.4 $\mathrm{mm}$ from the brain surface). A flap of skull was removed overlying the cortex above the left striatum, and a "well" surrounding the recording site was fashioned from dental cement. The well was covered with paraffin wax during recording, and the cisterna magna was punctured to reduce brain pulsations. For electroencephalogram (EEG) monitoring of cortical activity, a silver wire electrode was placed against the dura overlying the cortex $\sim 3 \mathrm{~mm}$ anterior to the intracellular recording site and secured in place with dental cement.

Intracellular recording and stimulation procedures. Intracellular records were made using micropipettes pulled from 3.0-mm-diameter glass and filled with $1 \mathrm{~m} \mathrm{~K}$-acetate containing $4 \%$ biocytin. Current-voltage relationships were obtained by injecting hyperpolarizing and depolarizing current pulses through the micropipette using an Axoclamp-2B amplifier (Axon Instruments, Foster City, CA.) configured in current-clamp mode. Postsynaptic potentials were evoked by single test stimulus pulses applied to the cortical electrode $(0.1 \mathrm{~Hz}, 0.1 \mathrm{msec}$ biphasic pulses, $300-$ $990 \mu \mathrm{A})$.

In plasticity experiments, responses to cortical test stimuli were routinely recorded for $20 \mathrm{~min}$ before and $20 \mathrm{~min}$ after HFS of the substantia nigra (HFS-SN) (six trains of 50 pulses at $100 \mathrm{~Hz}$ ). Each train was triggered from the same baseline membrane potential, and successive trains were separated by at least $10 \mathrm{sec}$. The effects of HFS of the cortex (HFS$\mathrm{Cx}$ ) (six trains of 50 pulses at $100 \mathrm{~Hz}$ ) and HFS-Cx paired with stimulation of the substantia nigra (HFS-Cx + SN) were also investigated. Stimulus pulses applied to the substantia nigra were biphasic, $0.5 \mathrm{msec}$ total duration, and $990 \mu \mathrm{A}$ amplitude, except in one experiment in which a current intensity $(190 \mu \mathrm{A})$ was used that had been shown before the acute experiment to support intracranial self-stimulation behavior in that animal (Reynolds et al., 2001). Stimulus trains were paired with a small depolarizing current injection $(0.20 \pm 0.16 \mathrm{nA}, 500 \mathrm{msec}$ duration) to ensure action potential firing throughout the trains.

Responses to cortical and substantia nigra test stimuli and current injection were acquired at predetermined membrane potentials using a locally constructed functional clamp (Reynolds and Wickens, 2003) to trigger data acquisition. As necessary, continuous hyperpolarizing current was injected through the micropipette to attenuate action potential firing and facilitate the measurement of subthreshold events. All of the waveform data were digitized at $10 \mathrm{kHz}$ by a Digidata 1200B (Axon Instruments), displayed using pClamp 8 software (Axon Instruments), and stored to disk (Pentium III personal computer). The analog membrane potential signal was also recorded on FM tape (model MR30; TEAC, Tokyo, Japan).

At the end of experiments, neurons were filled with biocytin by applying depolarizing current pulses $(0.8-1.5 \mathrm{nA}, 3 \mathrm{~Hz}, 10-15 \mathrm{~min})$. Vibratome sections $(50 \mu \mathrm{m})$ were processed using standard histological procedures (Horikawa and Armstrong, 1988), and labeled cells were identified by light microscopy. Stimulating electrode positions were verified in six experiments using either cresyl violet $(0.01 \%)$ or unstained sections. All of the substantia nigra electrode tips were situated within $500 \mu \mathrm{m}$ of the pars compacta (data not shown).

Analysis of cellular properties. Cellular properties were measured using Axograph 4.9 (Axon Instruments Inc.), and peristimulus spike firing was analyzed using Spike 2 (version 4.17; Cambridge Electronics Design, Cambridge, UK). Input resistance was determined from the slope of a regression line fitted to the membrane potential produced by a series of current pulses $(-0.2$ to $+0.2 \mathrm{nA})$. The amplitude of PSPs was defined as the difference between the baseline immediately preceding the stimulus artifact and the peak of the PSP. The duration of the f-AHP after a single action potential was measured at one-half f-AHP amplitude (Reynolds and Wickens, 2000). The prolonged AHP was defined as the longduration hyperpolarization after a depolarizing event (current pulse or cortical stimulus). The amplitude of the prolonged AHP was defined as the difference between the baseline membrane potential (10 msec before the event) and the maximum hyperpolarization. The duration was defined as the time between the onset of the prolonged AHP and return of the membrane potential to baseline (see Fig. 2A).

Analysis of membrane potential fluctuations. Membrane potential fluctuations were quantified by using all-amplitude histograms made from $30 \mathrm{sec}$ of membrane potential activity (Reynolds and Wickens, 2000) to display the time spent at any given membrane potential. A two-Gaussian distribution provided a satisfactory fit to the histograms, which were frequently skewed in the hyperpolarizing direction. The Gaussian distributions yielded the average hyperpolarized and depolarized membrane potentials ( $\mu 1$ and $\mu 2)$ and their respective SDs (s1 and s2). The amplitude of the membrane potential fluctuations was estimated by the difference between the average depolarized and hyperpolarized membrane potentials.

Electroencephalogram analysis. In eight experiments, the cortical EEG was recording simultaneously with the membrane potential waveform of the cholinergic interneurons. The EEG signal was amplified and filtered to pass signals in the $0.01-400 \mathrm{~Hz}$ band (model 74-20-1; Frederick Haer, Brunswick, ME) before digitizing at $10 \mathrm{kHz}$. All-amplitude histograms were made from the EEG waveform, and a two-Gaussian distribution was fitted to the data in a manner similar to that of the membrane potential fluctuations.

Statistical analysis. Data are summarized as mean \pm SD unless otherwise stated. The probability level for statistical significance was set at $p=0.05$.

\section{Results}

Intracellular recordings were obtained from a total of 14 cholinergic interneurons in 12 rats. Cholinergic interneurons had distinctive electrophysiological properties (Reynolds and Wickens, 2004). They fired in a tonic pattern (average firing rate, $3.2 \pm 1.7$ $\mathrm{Hz}$ ) (Fig. 1). Action potentials (half-duration, $0.78 \pm 0.17 \mathrm{msec}$ ) and the f-AHP (half-duration, $128 \pm 45 \mathrm{msec}$ ) were of relatively long duration. Hyperpolarizing current pulses produced an initial hyperpolarization followed by a "sag" in membrane potential, indicative of the presence of an $I_{\mathrm{h}}$ membrane conductance (Jiang and North, 1991; Kawaguchi, 1992). Of eight presumed cholinergic interneurons examined histologically, all of them had large fusiform or triangular somata, elongated processes, and smooth dendrites typical of cholinergic interneurons, as described previously (Bolam et al., 1984; Wilson et al., 1990; Kawaguchi, 1993).

\section{Prolonged afterhyperpolarization}

The injection of positive current through the recording micropipette induced repetitive spike firing in all eight of the cholinergic interneurons tested. After such an episode of spike firing, a prolonged membrane hyperpolarization was noted lasting 390-1400 msec, depending on the magnitude of current injection (Fig. 2A). This prolonged AHP was often of sufficient magnitude and duration to produce a pause in the tonic irregular firing pattern of 

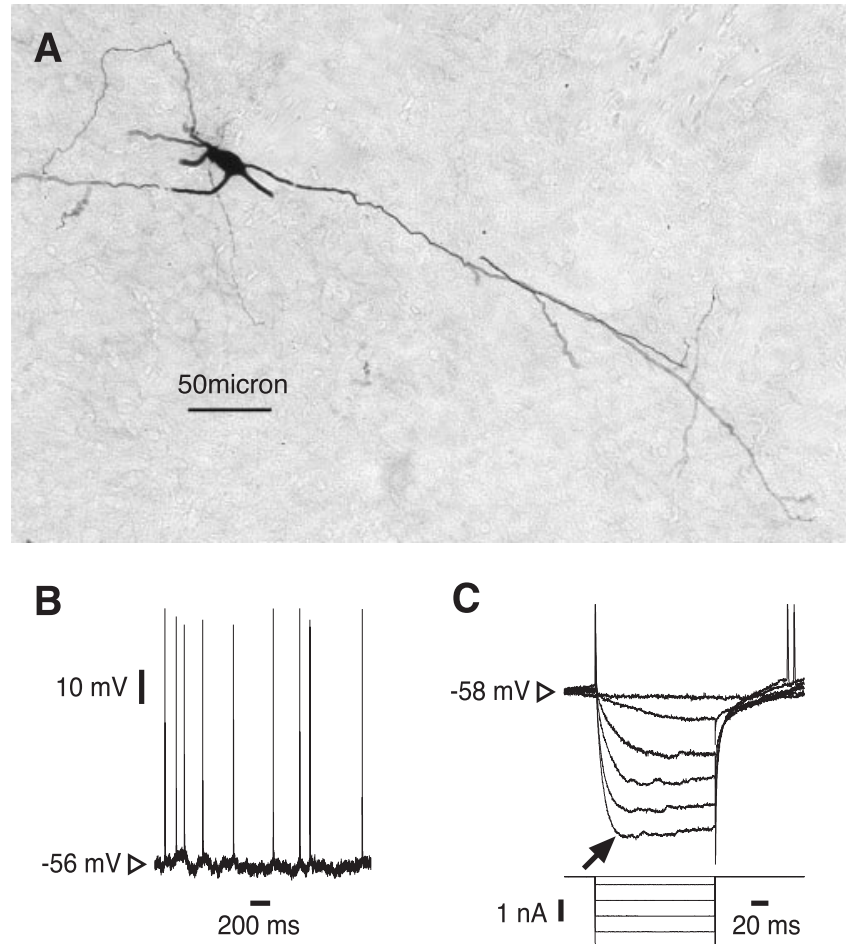

Figure 1. Morphology and electrophysiology of a cholinergic interneuron recorded intracellularly in vivo. $A$, Neuron identified as a cholinergic interneuron because of its relatively large soma and smooth aspiny dendrites. $B$, Recording of the membrane potential activity of the cholinergic interneuron shown in $A$. This interneuron was spontaneously active and fired action potentials in a tonic pattern. C, An initial membrane hyperpolarization followed by a characteristic depolarizing sag (arrow) was induced in response to the injection of hyperpolarizing current steps of large amplitude and a rebound depolarization and spike firing occurred at the end of the step. Each trace is the average of four responses; hence, action potentials are attenuated.

the cholinergic interneurons (Fig. $2 B$ ). In contrast, brief injections of hyperpolarizing current did not obviously interrupt the tonic activity of the cholinergic interneurons and were usually followed instead by a small rebound depolarization and a single spike.

The amplitude and duration of the prolonged AHP were highly correlated with the number of action potentials fired during a depolarizing current step $(r=-0.94$ and 0.98 , respectively; $p<0.001$ ), both in individual interneurons and in the pooled data (Fig. 3A). Significantly, the amplitude of the AHP also correlated with the degree of membrane depolarization measured midway through the step $(r=-0.98 ; p<0.05)$, suggesting that depolarization rather that action potentials per se may be the most important factor in determining the degree of hyperpolarization.

To further investigate this, we examined the effect of subthreshold depolarization from hyperpolarized holding potentials. With increasing depolarization above the holding level $(-65 \pm 1 \mathrm{mV})$, there was a trend of increasing amplitude and duration of the prolonged AHP (Fig. $3 B, C$ ). A similar hyperpolarization was also observed after synaptic PSPs evoked by activating cortical inputs. Single-pulse stimulation of the contralateral cerebral cortex at maximal intensity evoked a small depolarizing PSP (amplitude, $2.8 \pm 1.7 \mathrm{mV}$; duration, $92.5 \pm$ $56.5 \mathrm{msec}$ ), which was immediately followed by a hyperpolarization (Fig. 3D). This was seen in $20 \%$ of recordings before substantia nigra stimulation but was present in all of the neurons tested after substantia nigra HFS (see below). With the holding current removed, a cortically evoked response of this kind was
A

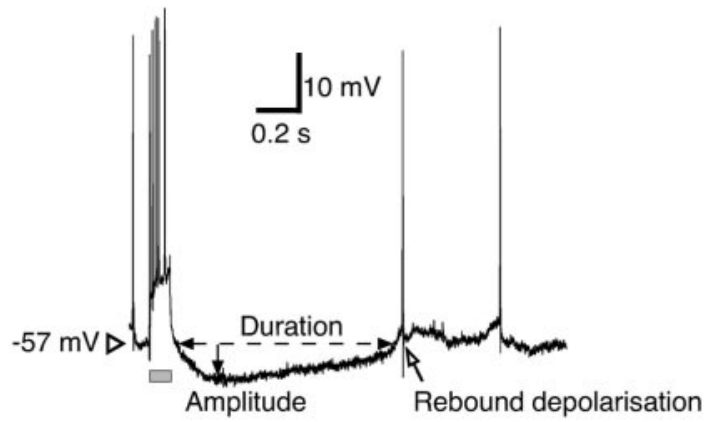

B

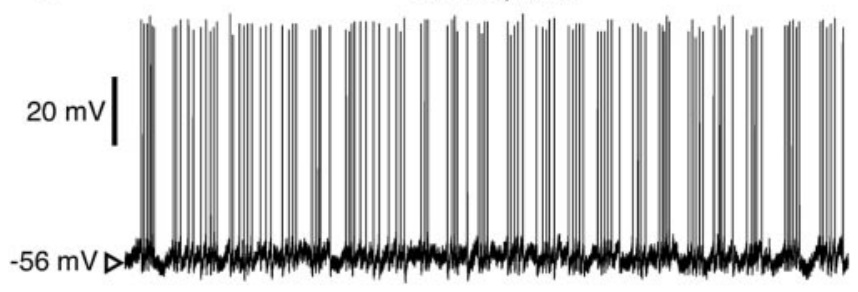

$1 \mathrm{~s}$

Test period

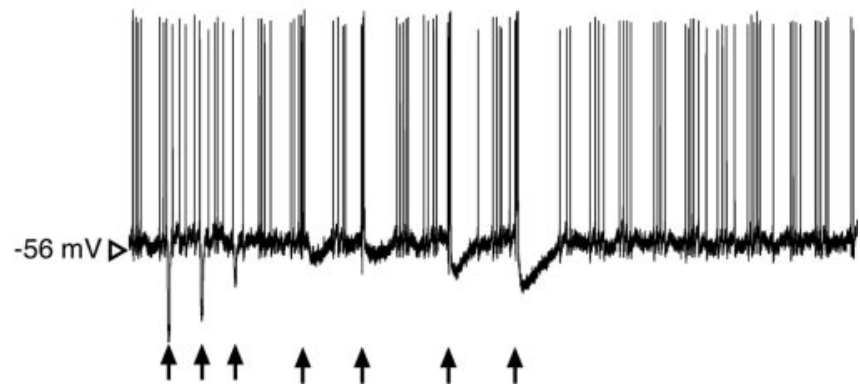

Figure 2. A prolonged AHP interrupts the tonic firing of cholinergic interneurons. A, Suprathreshold current injection (gray bar) induced action potential firing and a subsequent hyperpolarization followed by rebound depolarization and a single spike. $B$, The firing pattern of a cholinergic interneuron (top) was interrupted by a current injection series (bottom). Prolonged AHPs elicited by a 90 msec duration depolarizing current injection $(0.2,0.4,0.6,0.8 \mathrm{nA}$; last 4 arrows) induced pauses in tonic firing that were not seen after hyperpolarizing pulses $(-0.6$, $-0.4,-0.2 \mathrm{nA}$; first 3 arrows) of similar duration.

associated with a period of reduced action potential firing after the stimulus pulse (Fig. 3E).

Together, the above data show that brief depolarization can elicit a prolonged AHP. The amplitude of this AHP is related to the extent of action potential firing, but, importantly, a hyperpolarization of between 100 and $200 \mathrm{msec}$ duration also occurs after subthreshold depolarization and single subthreshold PSPs. The AHP interrupts tonic firing of the cells and thus could be a mechanism for the pause response seen in behaving animals.

\section{Effects of plasticity-inducing stimulation}

If the prolonged AHP is involved in pause responses observed during behavioral conditioning, then it should show plasticity in response to learning-related stimuli. Activation of the nigrostriatal dopamine pathway is a prominent feature under such conditions during behavior (Schultz, 1998; Hyland et al., 2002) and has well characterized effects on plasticity in other components of the striatum (Calabresi et al., 1992; Kerr and Wickens, 2001; Reynolds et al., 2001; Tang et al., 2001). We therefore measured the effect of plasticity-inducing high-frequency stimulation of 
A
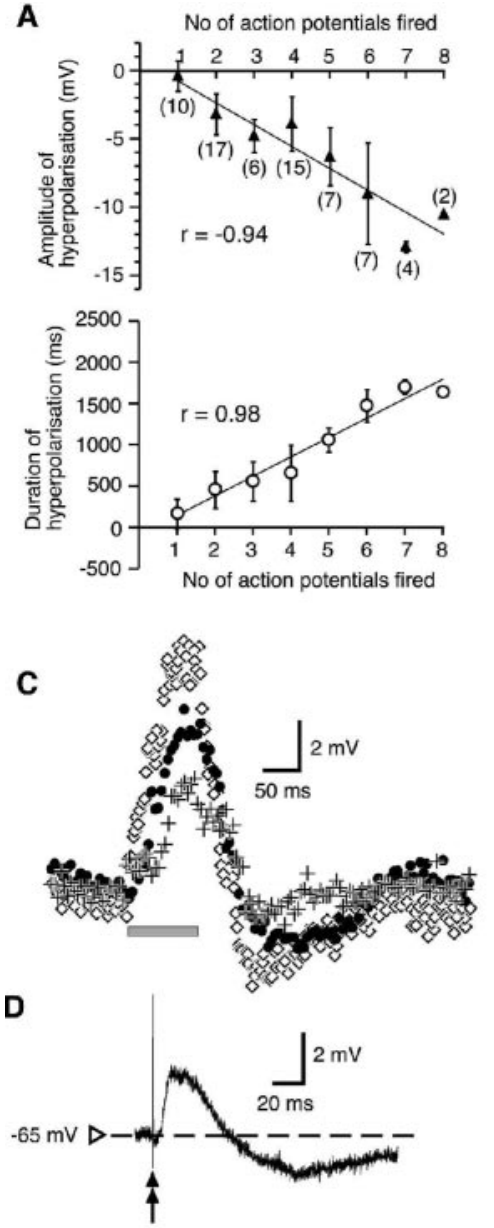

B
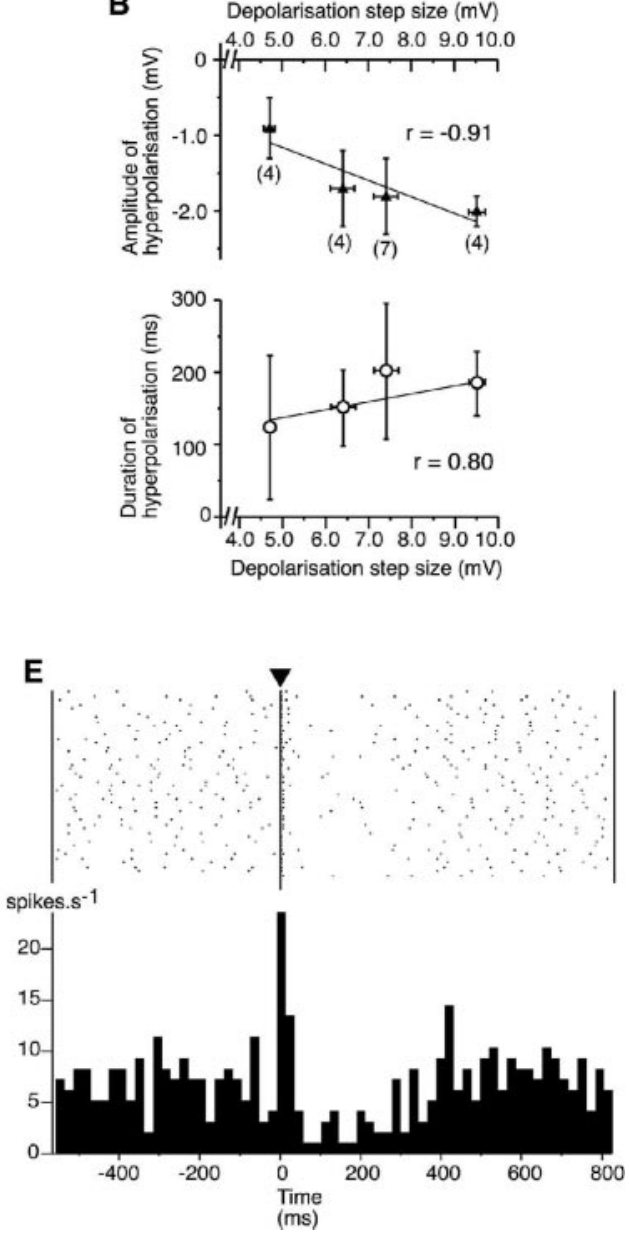

Figure 3. Relationship between the magnitude of depolarizing events and the prolonged AHP. A, Both the amplitude and duration of the prolonged AHP were correlated with the number of action potentials fired from a suprathreshold depolarizing step. The number of measurements at each point in the graphs is in parentheses. $B$, Action potential firing is not essential for the prolonged AHP: subthreshold depolarization in response to small-amplitude current injection (gray bar in C) induces a subsequent hyperpolarization that is dependent on the level of depolarization obtained ( $B, C)$. Data obtained from two neurons are as follows: effects of step size 4.7 (crosses), 6.4 (filled circles), and $9.5 \mathrm{mV}$ (open diamonds) measured in neuron 1; effects of step size $7.4 \mathrm{mV}$ measured in neuron 2. D, Depolarization in response to cortical stimulation (double-headed arrow) also induces a prolonged hyperpolarization (PSP average of 6 responses). $E$, A cortical response similar to that shown in $D$ induced a pause in tonic firing in a different neuron. Each line in the raster (top) represents a single sweep aligned to the time of the cortical stimulus (arrowhead) with each spike depicted by a dot. Note that the cortical PSP elicited one or two spikes per sweep. The histogram (bottom) shows the firing rate of the neuron summed across sweeps ( $25 \mathrm{msec}$ bin width). Note the reduction in firing between 100 and $300 \mathrm{msec}$ after the stimulus and the rebound firing at $400 \mathrm{msec}$.

the substantia nigra using parameters known to be behaviorally rewarding (Reynolds et al., 2001).

In all three of the identified cholinergic interneurons tested, substantia nigra stimulus trains induced an immediate depolarization of long duration $(15.9 \pm 6.5 \mathrm{sec})$ with peak $16.8 \pm 7.3 \mathrm{mV}$ above baseline (Fig. 4). Immediately after the stimulus trains, spontaneous hyperpolarizations became apparent (Fig. $4 \mathrm{~A}$, unfilled arrow). The hyperpolarizations were associated with short pauses in tonic firing and were correlated with the synchronized slow-wave activity of the cortical EEG (Fig. 4C). These hyperpolarizations were consistent with the occurrence of spontaneous AHPs and were apparent in the all-amplitude histograms of all three of the interneurons $20 \mathrm{~min}$ after stimulation (Fig. 5). A conspicuous increase in the range of small-amplitude membrane potential fluctuations in the hyperpolarizing direction is evident in Figure $5 B$ (open arrow). This is attributable to the AHPs.

Potentiation of excitatory inputs is a possible basis for the emergence of AHPs after HFS-SN. In two of three cholinergic interneurons, HFS-SN induced potentiation of PSP amplitude ( +14 and $+29 \%)$. In the third interneuron, potentiation $(+31 \%)$ was induced after substantia nigra stimulation was paired with HFS of the cortical input. Consistent with these changes, a more subtle increase in the range of membrane potential fluctuations in the depolarizing direction is evident in Figure $5 B$ (filled arrow). There were no associated changes in the cortical EEG waveform (Figs. $4 C, 5 C, D$ ), suggesting that substantia nigra stimulation did not modify the source of spontaneous afferent input that influences the membrane potential of striatal neurons under anesthesia (Tseng et al., 2001; Kasanetz et al., 2002; Reynolds and Wickens, 2004). In addition, changes in PSP amplitude were not associated with changes in input resistance (baseline, $27.0 \pm 7.2 \mathrm{M} \Omega ;+20 \mathrm{~min}, 24.1 \pm 6.4 \mathrm{M} \Omega$; $n=3)$. Thus, the increased PSPs and depolarizing membrane potential fluctuations may reflect increased synaptic efficacy of excitatory inputs to cholinergic interneurons.

Comparison of PSP traces before and after substantia nigra stimulation revealed the appearance of a hyperpolarization after the PSP. In all three of the interneurons, this AHP was not observed during baseline recordings but was present only after potentiation was induced (Fig. 6). A trend was apparent in the relationship between the degree of potentiation induced and the amplitude of this AHP: the higher the potentiation induced, the larger the amplitude of the hyperpolarization after the PSP.

Together, the above data indicate that stimulation of the substantia nigra induces the appearance of prolonged AHPs as a result of plastic changes in the efficacy of depolarizing synaptic inputs.

\section{Discussion}

Based on in vivo intracellular recordings from the rare cholinergic interneurons, we describe a depolarization-dependent prolonged AHP. Although AHPs have been described after action potential firing in vitro (Bennett et al., 2000; Suzuki et al., 2001; Pisani et al., 2002), our finding that subthreshold events can trigger an AHP is novel. We also found that potentiation of excitatory inputs to cholinergic interneurons led to facilitation of the prolonged AHPs evoked by these inputs. The magnitude of the facilitation was positively related to the degree of potentiation of excitatory inputs. Furthermore, AHPs evoked experimentally caused pauses in the characteristic in vivo firing activity of the interneurons. Thus, the acquisition of these pauses through plasticity of excitatory inputs may be related to changes in pause responses that occur in TANs during learning. 

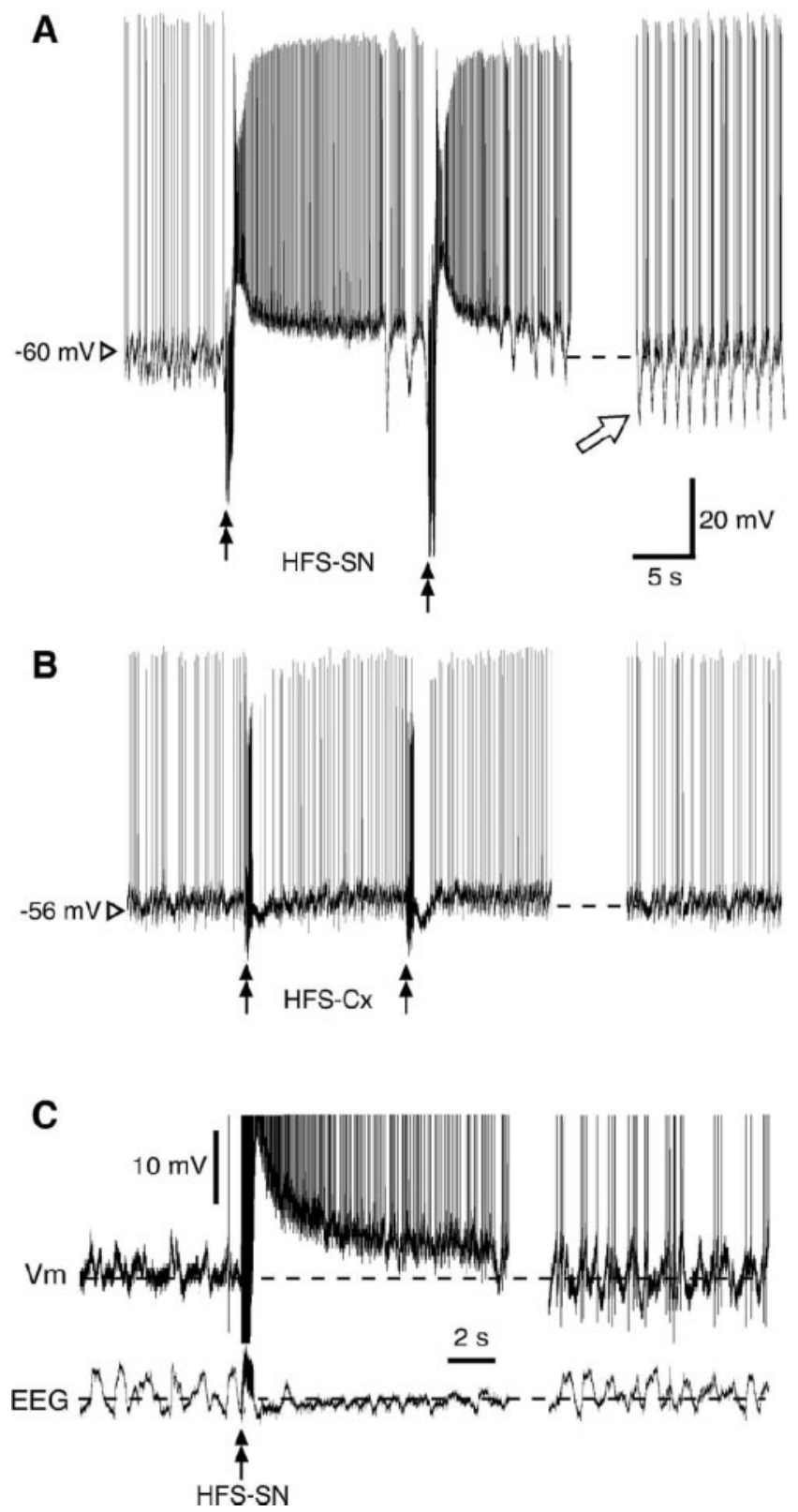

Figure 4. Substantia nigra stimulation induced membrane depolarization and lasting spontaneous membrane hyperpolarizations in cholinergic interneurons. $A$, High-frequency stimulation applied to the substantia nigra (double arrows) induced a large-amplitude membrane depolarization of long duration. One minute after application of the last of six stimulus trains, after recovery from the depolarization, spontaneous hyperpolarizations were present (right trace), which persisted for many minutes after the application of the stimulus trains. $B$, Neither the stimulus-induced depolarizations nor the spontaneous hyperpolarizations were observed in a different cholinergic interneuron after HFS of the cortex. Instead, a prolonged AHP was seen after the application of each stimulus train (double arrows). C, Augmented spontaneous membrane potential fluctuations after HFS of the substantia nigra are correlated with the EEG. A high-gain trace of the membrane potential $(\mathrm{Vm})$ of another cholinergic interneuron was recorded simultaneously with the cortical EEG before and during HFS-SN (left traces) and 1 min after the six stimulus trains (right traces). The membrane potential fluctuations and the EEG waveform are correlated before the application of the stimulus trains, as described previously (Reynolds and Wickens, 2004). The prolonged depolarization in Vm after each stimulus train (double arrow) was accompanied by a transient desynchronization of the EEG. Note the increased amplitude of subthreshold fluctuations in Vm after the stimulus trains, when the EEG waveform had reverted to synchronized slow-wave activity (right traces). The fluctuations are punctuated by spontaneous hyperpolarizations below the original resting potential $(-64 \mathrm{mV}$; dotted line) and underlie the initiation of bursts of spontaneous action potential firing. These bursts are closely associated with fluctuations in the EEG waveform. Action potentials and stimulus artifact in Vm are truncated for clarity.
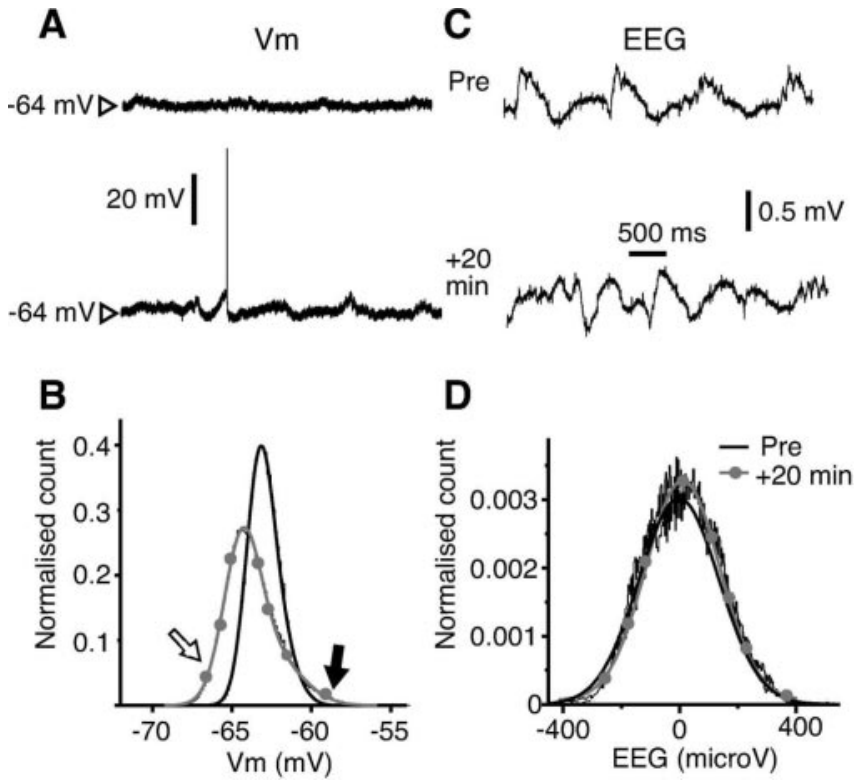

Figure 5. Substantia nigra stimulation induced lasting changes in subthreshold membrane potential activity. $A$, The amplitude of membrane potential fluctuations of cholinergic interneurons increased after substantia nigra stimulation and persisted at 20 min after the stimulus trains (+20 min). In this particular interneuron, spontaneous action potential firing was prevented by hyperpolarizing current injection (Pre) but recommenced after the stimulus trains. $B$ The emergence of spontaneous hyperpolarizations (unfilled arrow) and an increase in the range of depolarized membrane potentials (filled arrow) were apparent in the all-amplitudes histogram of membrane potential activity at $+20 \mathrm{~min}$ (gray trace with circles) when compared with baseline activity (black trace). C, There were no associated changes in the simultaneously recorded cortical EEG waveform or the all-amplitudes histograms of EEG activity after substantia nigra stimulation $(D)$.

The present findings are consistent with previous studies showing that cholinergic interneurons have an intrinsic mechanism for generating prolonged AHPs. The prolonged AHP we describe in vivo is similar in time course and amplitude to AHPs described in vitro. These in vitro studies suggest that the prolonged AHP is unlikely to require calcium because it remains intact after calcium depletion and blockade of small-conductance calcium-activated potassium channels (Bennett et al., 2000). A likely alternative is that the AHP is mediated by the activation of an A-type voltage-dependent potassium channel (Song et al., 1998). Although the mechanism of the AHP remains to be determined, the present results show that plasticity of the pause response may be achieved by modulation of the excitatory inputs to cholinergic interneurons. Furthermore, our observation that the prolonged AHP was triggered by subthreshold stimulation is consistent with the observation in monkeys that the pause response may occur in the absence of a preceding burst of action potentials (Aosaki et al., 1994a; Apicella et al., 1997; Watanabe and Kimura, 1998).

Although our results suggest that the potentiation of excitatory inputs is necessary for an AHP after cortical stimulation, in $20 \%$ of cholinergic interneurons a hyperpolarizing response was present before plasticity was induced (e.g., Fig. 3D). One compelling explanation for this is that corticostriatal synapses onto this subset of interneurons were already potentiated at baseline, analogous to the $17 \%$ of TANs in behaving animal experiments that exhibit a pause response to a cue before conditioning (Aosaki et al., 1995). However, although the hyperpolarizing component of the cortically evoked response exhibited a similar time course to an intrinsically generated AHP (Fig. 3C,D), its peak amplitude 

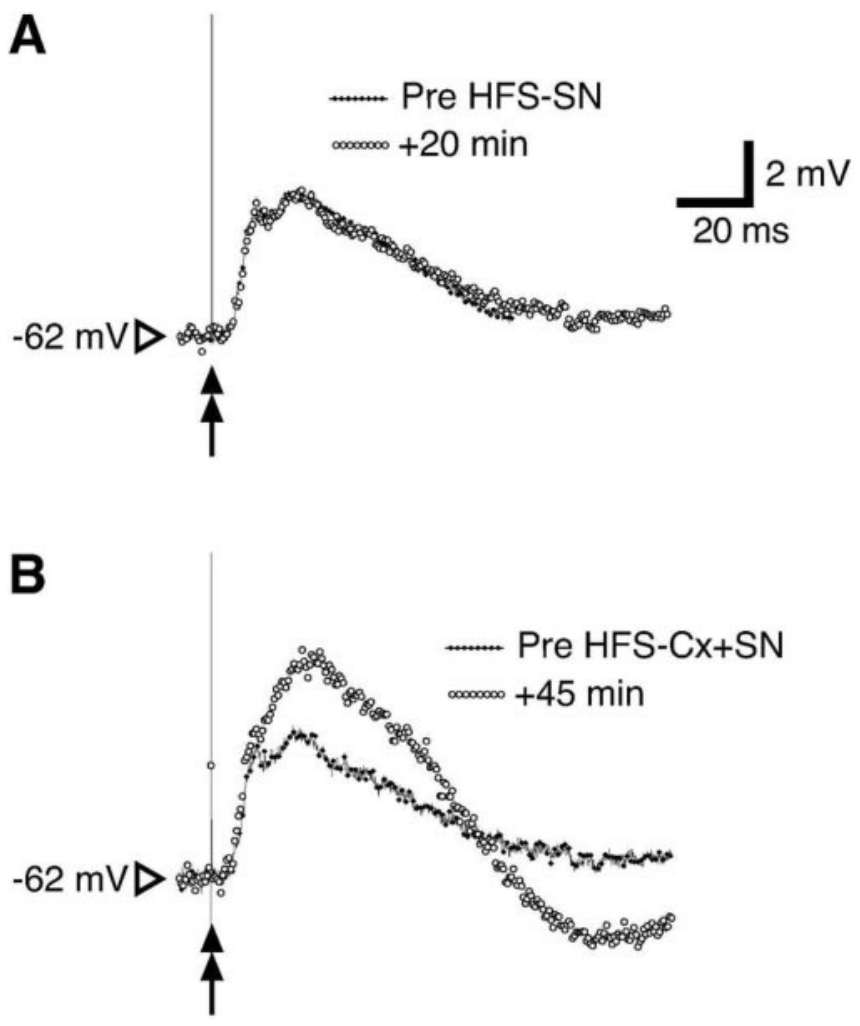

Figure 6. Potentiation of PSPs induced by substantia nigra stimulation was associated with a cortically evoked prolonged AHP. A, Postsynaptic potentials evoked in one cholinergic interneuron were not potentiated $20 \mathrm{~min}$ after HFS-SN. Note that there was no discernible hyperpolarization after the PSP, because the PSP amplitude remained at baseline levels. B, Persistent potentiation was induced in the same interneuron after HFS-Cx+SN. Comparison of the PSPs just before (Pre) HFS-Cx+SN (same as the +20 min trace in $A$ ) and 45 min after shows that corticostriatal potentiation was associated with the appearance of an AHP subsequent to the depolarizing component of the PSP. All of the PSP traces are the average of at least six PSPs recorded over $1 \mathrm{~min}$.

was substantially larger than that predicted from the preceding depolarization (Fig. 3B). This suggests that the baseline response may have included an IPSP simultaneously induced by cortical stimulation. This is likely to be disynaptic (Suzuki et al., 2001) and mediated via presumed synaptic contacts between GABAergic neurons and cholinergic interneurons (Chang and Kita, 1992; Bennett and Wilson, 1998). In particular, parvalbumin-positive GABAergic interneurons have a dense cortical innervation, exhibit a relatively low threshold to excitatory stimuli, and exert a strong inhibitory influence on neighboring neurons (Lapper et al., 1992; Kawaguchi et al., 1995; Parthasarathy and Graybiel, 1997; Koos and Tepper, 1999).

It was not possible to determine the degree to which synaptic inhibition contributed to the cortically evoked hyperpolarization either at baseline or after the excitatory responses were potentiated. Because our recordings were obtained serendipitously, we did not design the experiments to test the effect of blocking inhibitory neurotransmission. In addition, sufficiently hyperpolarizing the cell membrane of cholinergic interneurons to test the voltage dependence of the prolonged AHP was difficult without destabilizing the recording. Thus, the contribution of IPSPs to enhanced hyperpolarizations induced either by cortical stimulation or spontaneously after substantia nigra stimulation could not be ruled out in our in vivo model.

Despite the possible influence of IPSPs on the amplitude of the cortically evoked hyperpolarization, a number of previous obser- vations led us to believe that a depolarization-induced AHP may be the major determinant of its duration. Stimulation of the ipsilateral cortex readily induces EPSPs in striatal GABAergic interneurons in vitro (Kawaguchi, 1993) and in vivo (Kita, 1993); however, firing thus evoked is usually limited to single action potentials elicited at short latency $(<10 \mathrm{msec})$. Hence, the inhibition induced in target neurons is likely to be relatively brief in comparison with the duration of the intrinsic AHP. In support of this, we recently reported disynaptic IPSPs of short latency (6 msec to peak) and duration (20 msec maximum) in cholinergic interneurons after activation of the ipsilateral corticostriatal projection (Reynolds and Wickens, 2004). Thus, IPSPs evoked in cholinergic interneurons are likely to influence only the initial depolarizing and early hyperpolarizing components of the simultaneously evoked cortical response. Although an IPSP might increase the peak amplitude of the AHP as postulated above, partial shunting of the initial excitatory component of the cortical response may, paradoxically, lead to a slight reduction in the duration of the AHP by reducing the effective membrane depolarization. Consequently, after concomitant excitation of cholinergic and GABAergic interneurons, the period of induced hyperpolarization is likely to be similar or less than that obtained purely by an excitation-induced AHP. This is also relevant to the spontaneous hyperpolarizations and enhanced PSPs that occur after potentiation is induced because enhanced cortical input is more likely to result in coactivation of both cholinergic and GABAergic interneurons.

Potentiation of excitatory inputs to cholinergic interneurons was brought about by substantia nigra stimulation in the present study. This potentiation is probably dopamine-dependent because potentiation of excitatory inputs to cholinergic interneurons has recently been reported by Suzuki et al. (2001) to require dopamine $D_{1} / D_{5}$ receptor activation. Similarly, dopamine has been found to be necessary for synaptic plasticity in spiny projection neurons (Wickens et al., 1996; Calabresi et al., 2000). It is, however, known that stimulation of the substantia nigra also activates cortical neurons that pass through the brainstem, inducing an axon reflex in their collaterals that innervate the striatum (Wilson et al., 1982). Thus, the membrane depolarization we report during HFS of the substantia nigra probably reflects simultaneous activation of cortical and nigral inputs to cholinergic interneurons. Although dopamine neurons are difficult to activate electrically, we are confident that our stimulus trains would have released dopamine for a number of reasons. First, we selected stimulus parameters and used electrode placements that favor the activation of dopaminergic elements (Yeomans, 1989), and such stimulation has been shown to induce a measurable electrochemical dopamine signal over a widespread area of the striatum (Garris et al., 1997). Second, the activation of dopamine $\mathrm{D}_{5}$ receptors in vitro induces effects on cholinergic interneurons similar to those we observed during substantia nigra stimulation (Aosaki et al., 1998; Centonze et al., 2003). Finally, the plasticityinducing stimulation used in the present study was the same as that shown to induce dopamine $\mathrm{D}_{1} / \mathrm{D}_{5}$ receptor-dependent potentiation in vivo in other striatal elements receiving input from the corticostriatal pathway (Reynolds et al., 2001). Thus, although we were unable to confirm the direct involvement of dopamine in the potentiation induced by substantia nigra stimulation, a number of previous observations support its involvement.

The role of dopamine in the plasticity of excitatory inputs to cholinergic interneurons is relevant to the mechanism of the pause responses in behaving animals. We showed previously that 
dopamine depletion reduces the amplitude of spontaneous postsynaptic potentials recorded in vivo (Reynolds and Wickens, 2000). Thus, dopamine-dependent potentiation of corticostriatal inputs by substantia nigra stimulation is likely to lead to facilitation of prolonged AHPs and the appearance of pause responses, whereas dopamine depletion is likely to result in depolarizing events of insufficient amplitude to trigger the prolonged AHP. These properties are consistent with the dopamine-dependent nature of the learning-related pause response obtained in awake animals (Aosaki et al., 1994b; Watanabe and Kimura, 1998).

Under behavioral conditions, reward-related learning is associated with dopamine cell burst firing in response to rewards and signals predicting rewards (Mirenowicz and Schultz, 1996; Schultz et al., 1998). We hypothesize that this would lead to potentiation of excitatory inputs to cholinergic interneurons, so that these would produce sufficient depolarization to trigger the prolonged AHP and, hence, the pause response. There are several features of the prolonged AHP that are consistent with this hypothesis. The latency to onset of the prolonged AHP (immediately after the cortical PSP of $\sim 90 \mathrm{msec}$ average duration) is comparable with the latency of the pause response in TANs (60$125 \mathrm{msec}$ ) (Aosaki et al., 1994b; Matsumoto et al., 2001). Furthermore, the duration of the prolonged AHP (100-200 msec) (Fig. $3 B$ ) is comparable with the duration of the pause response recorded in awake animals (130-250 msec) (Watanabe and Kimura, 1998; Matsumoto et al., 2001). Finally, the gradual time course of acquisition of the pause response (Aosaki et al., 1994a, their Fig. 10) is consistent with the progressive augmentation of spontaneous hyperpolarizations over a series of substantia nigra stimulus trains (Fig. 4A). Together, these observations suggest that changes in the prolonged AHPs secondary to synaptic plasticity in excitatory inputs may underlie the acquisition of pause responses in TANs in awake animals during learning of behavioral responses.

\section{References}

Aosaki T, Tsubokawa H, Ishida A, Watanabe K, Graybiel AM, Kimura M (1994a) Responses of tonically active neurons in the primate's striatum undergo systematic changes during behavioral sensorimotor conditioning. J Neurosci 14:3969-3984.

Aosaki T, Graybiel AM, Kimura M (1994b) Effect of the nigrostriatal dopamine system on acquired neural responses in the striatum of behaving monkeys. Science 265:412-415.

Aosaki T, Kimura M, Graybiel AM (1995) Temporal and spatial characteristics of tonically active neurons of the primate's striatum. J Neurophysiol 73:1234-1252.

Aosaki T, Kiuchi K, Kawaguchi Y (1998) Dopamine $\mathrm{D}_{1}$-like receptor activation excites rat striatal large aspiny neurons in vitro. J Neurosci 18:5180-5190.

Apicella P, Scarnati E, Schultz W (1991) Tonically discharging neurons of monkey striatum respond to preparatory and rewarding stimuli. Exp Brain Res 84:672-675.

Apicella P, Legallet E, Trouche E (1997) Responses of tonically discharging neurons in the monkey striatum to primary rewards delivered during different behavioral states. Exp Brain Res 116:456-466.

Bennett BD, Wilson CJ (1998) Synaptic regulation of action potential timing in neostriatal cholinergic interneurons. J Neurosci 18:8539-8549.

Bennett BD, Callaway JC, Wilson CJ (2000) Intrinsic membrane properties underlying spontaneous tonic firing in neostriatal cholinergic interneurons. J Neurosci 20:8493-8503.

Bishop GA, Chang HT, Kitai ST (1982) Morphological and physiological properties of neostriatal neurons: an intracellular horseradish peroxidase study in the rat. Neuroscience 7:179-191.

Bolam JP, Wainer BH, Smith AD (1984) Characterization of cholinergic neurons in the rat neostriatum. A combination of choline acetyltransferase immunocytochemistry, Golgi-impregnation and electron microscopy. Neuroscience 12:711-718.
Calabresi P, Maj R, Pisani A, Mercuri NB, Bernardi G (1992) Long-term synaptic depression in the striatum: physiological and pharmacological characterization. J Neurosci 12:4224-4233.

Calabresi P, Gubellini P, Centonze D, Picconi B, Bernardi G, Chergui K, Svenningsson P, Fienberg AA, Greengard P (2000) Dopamine and cAMP-regulated phosphoprotein $32 \mathrm{kDa}$ controls both striatal long-term depression and long-term potentiation, opposing forms of synaptic plasticity. J Neurosci 20:8443-8451.

Centonze D, Grande C, Usiello A, Gubellini P, Erbs E, Martin A, Pisani A, Tognazzi N, Bernardi G, Moratalla R, Borrelli E, Calabresi P (2003) Receptor subtypes involved in the presynaptic and postsynaptic actions of dopamine on striatal interneurons. J Neurosci 23:6245-6254.

Chang HT, Kita H (1992) Interneurons in the rat striatum: relationships between parvalbumin neurons and cholinergic neurons. Brain Res 574:307-311.

Garris PA, Christensen JR, Rebec GV, Wightman RM (1997) Real-time measurement of electrically evoked extracellular dopamine in the striatum of freely moving rats. J Neurochem 68:152-161.

Horikawa K, Armstrong WE (1988) A versatile means of intracellular labeling: injection of biocytin and its detection with avidin conjugates. J Neurosci Methods 25:1-11.

Hyland BI, Reynolds JN, Hay J, Perk CG, Miller R (2002) Firing modes of midbrain dopamine cells in the freely moving rat. Neuroscience 114:475-492.

Jiang ZG, North RA (1991) Membrane properties and synaptic responses of rat striatal neurones in vitro. J Physiol (Lond) 443:533-553.

Kasanetz F, Riquelme LA, Murer MG (2002) Disruption of the two-state membrane potential of striatal neurones during cortical desynchronisation in anaesthetised rats. J Physiol (Lond) 543:577-589.

Kawaguchi Y (1992) Large aspiny cells in the matrix of the rat neostriatum in vitro: physiological identification, relation to the compartments and excitatory postsynaptic currents. J Neurophysiol 67:1669-1682.

Kawaguchi Y (1993) Physiological, morphological, and histochemical characterization of three classes of interneurons in rat neostriatum. J Neurosci 13:4908-4923.

Kawaguchi Y, Wilson CJ, Augood SJ, Emson PC (1995) Striatal interneurones: chemical, physiological and morphological characterization. Trends Neurosci 18:527-535.

Kerr JN, Wickens JR (2001) Dopamine D-1/D-5 receptor activation is required for long-term potentiation in the rat neostriatum in vitro. J Neurophysiol 85:117-124.

Kimura M, Rajkowski J, Evarts E (1984) Tonically discharging putamen neurons exhibit set-dependent responses. Proc Natl Acad Sci USA 81:4998-5001.

Kita H (1993) GABAergic circuits of the striatum. Prog Brain Res 99:51-72.

Koos T, Tepper JM (1999) Inhibitory control of neostriatal projection neurons by GABAergic interneurons. Nat Neurosci 2:467-472.

Lapper SR, Smith Y, Sadikot AF, Parent A, Bolam JP (1992) Cortical input to parvalbumin-immunoreactive neurones in the putamen of the squirrel monkey. Brain Res 580:215-224.

Matsumoto N, Minamimoto T, Graybiel AM, Kimura M (2001) Neurons in the thalamic CM-Pf complex supply striatal neurons with information about behaviorally significant sensory events. J Neurophysiol 85:960-976.

Mirenowicz J, Schultz W (1996) Preferential activation of midbrain dopamine neurons by appetitive rather than aversive stimuli. Nature 379:449-451.

Morris G, Arkadir D, Nevet A, Vaadia E, Bergman H (2004) Coincident but distinct messages of midbrain dopamine and striatal tonically active neurons. Neuron 43:133-143.

Parthasarathy HB, Graybiel AM (1997) Cortically driven immediate-early gene expression reflects modular influence of sensorimotor cortex on identified striatal neurons in the squirrel monkey. J Neurosci 17:2477-2491.

Pisani A, Bonsi P, Catania MV, Giuffrida R, Morari M, Marti M, Centonze D, Bernardi G, Kingston AE, Calabresi P (2002) Metabotropic glutamate 2 receptors modulate synaptic inputs and calcium signals in striatal cholinergic interneurons. J Neurosci 22:6176-6185.

Ravel S, Legallet E, Apicella P (1999) Tonically active neurons in the monkey striatum do not preferentially respond to appetitive stimuli. Exp Brain Res 128:531-534.

Reynolds JNJ, Wickens JR (2000) Substantia nigra dopamine regulates syn- 
aptic plasticity and membrane potential fluctuations in the rat neostriatum, in vivo. Neuroscience 99:199-203.

Reynolds JNJ, Wickens JR (2003) A state-dependent trigger for electrophysiological recording at predetermined membrane potentials. J Neurosci Methods 131:111-119.

Reynolds JNJ, Wickens JR (2004) The corticostriatal input to giant aspiny interneurons in the rat: a candidate pathway for synchronising the response to reward-related cues. Brain Res 1011:115-128.

Reynolds JNJ, Hyland BI, Wickens JR (2001) A cellular mechanism of reward-related learning. Nature 413:67-70.

Schultz W (1998) Predictive reward signal of dopamine neurons. J Neurophysiol 80:1-27.

Schultz W, Tremblay L, Hollerman JR (1998) Reward prediction in primate basal ganglia and frontal cortex. Neuropharmacology 37:421-429.

Song WJ, Tkatch T, Baranauskas G, Ichinohe N, Kitai ST, Surmeier DJ (1998) Somatodendritic depolarization-activated potassium currents in rat neostriatal cholinergic interneurons are predominantly of the A type and attributable to coexpression of Kv4.2 and Kv4.1 subunits. J Neurosci $18: 3124-3137$.

Suzuki T, Miura M, Nishimura K, Aosaki T (2001) Dopamine-dependent synaptic plasticity in the striatal cholinergic interneurons. J Neurosci 21:6492-6501.

Tang K, Low MJ, Grandy DK, Lovinger DM (2001) Dopamine-dependent synaptic plasticity in striatum during in vivo development. Proc Natl Acad Sci USA 98:1255-1260.

Tseng KY, Kasanetz F, Kargieman L, Riquelme LA, Murer MG (2001) Cortical slow oscillatory activity is reflected in the membrane potential and spike trains of striatal neurons in rats with chronic nigrostriatal lesions. J Neurosci 21:6430-6439.

Ungless MA, Magill PJ, Bolam JP (2004) Uniform inhibition of dopamine neurons in the ventral tegmental area by aversive stimuli. Science 303:2040-2042.

Watanabe K, Kimura M (1998) Dopamine receptor-mediated mechanisms involved in the expression of learned activity of primate striatal neurons. J Neurophysiol 79:2568-2580.

Wickens JR, Begg AJ, Arbuthnott GW (1996) Dopamine reverses the depression of rat corticostriatal synapses which normally follows highfrequency stimulation of cortex in vitro. Neuroscience 70:1-5.

Wilson CJ, Chang HT, Kitai ST (1982) Origins of postsynaptic potentials evoked in identified rat neostriatal neurons by stimulation in substantia nigra. Exp Brain Res 45:157-167.

Wilson CJ, Chang HT, Kitai ST (1990) Firing patterns and synaptic potentials of identified giant aspiny interneurons in the rat neostriatum. J Neurosci 10:508-519.

Yeomans JS (1989) Two substrates for medial forebrain bundle self-stimulation: myelinated axons and dopamine axons. Neurosci Biobehav Rev 13:91-98. 